\title{
The centrifugal instability of the boundary-layer flow over slender rotating cones
}

\author{
Z. Hussain ${ }^{1}$, S. J. Garrett ${ }^{1}$ AND S. O. Stephen ${ }^{2}$ \\ ${ }^{1}$ Department of Mathematics, University of Leicester, Leicester LE1 7RH, UK \\ ${ }^{2}$ School of Mathematics, University of New South Wales, Sydney NSW 2052, Australia
}

(Received 21 July 2014)

Existing experimental and theoretical studies are discussed which lead to the clear hypothesis of a hitherto unidentified convective instability mode that dominates within the boundary-layer flow over slender rotating cones. The mode manifests as Görtlertype counter-rotating spiral vortices, indicative of a centrifugal mechanism. Although a formulation consistent with the classic rotating-disk problem has been successful in predicting the stability characteristics over broad cones, it is unable to identify such a centrifugal mode as the half-angle is reduced. An alternative formulation is developed and the governing equations solved using both short-wavelength asymptotic and numerical approaches to independently identify the centrifugal mode.

\section{Introduction}

This paper describes recent advances in the study of boundary-layer transition over rotating cones. In particular, we are concerned with the distinct convective instability mechanisms that dominate within the boundary layers over slender and broad rotating cones.

Our general interest in rotating cones is motivated by the flow around nose cones in aeroengine and spinning projectile applications. Here laminar-turbulent transition within the boundary layer can lead to significant increases in drag which has negative implications for fuel efficiency and control. Alternatively, turbulent flow can be encouraged as a means of heat transfer in situations where unwanted heat is generated, for example in re-entry. In any event, a complete understanding of the transition of such flows could lead to modifications in design and significant cost savings in aerospace technologies. It is clear that the linear-stability analyses presented here for cones rotating within incompressible and otherwise still fluids are of limited direct relevance in terms of these motivating applications. However, previous studies including Garrett et al. (2010), Hussain (2010), Hussain et al. (2011) have shown that there is a close link between the rotating disk and cone problems in still fluid and axial flow. Hence, the current still fluid study forms an important stepping stone to analysing the more complex problem where axial flow is introduced. More generally, this work should be considered as a further step towards fully classifying the instability mechanics within the global class of boundary layer flows over rotating bodies. Indeed, studies of the effects of enforced axial flow over broad cones and disks have already been published, Garrett \& Peake (2007), Garrett et al. (2010), Hussain (2010), Hussain et al. (2011), Towers \& Garrett (2013a,b).

The paper is structured as follows: we begin by summarizing the motivation for the 
hypothesis of an alternative instability mode in $\S 2$. In $\S 3$ the problem is formulated from the perspective of the asymptotic analysis of $\S 4$ and any variations in the formulation required by the numerical analysis are described in $\S 5$. The results of the two analyses are compared in $\S 6$ and conclusions drawn in $\S 7$.

\section{Motivating the hypothesis of an alternative mode}

The hypothesis of an alternative instability mode that is dominant on slender cones is justified by the consideration of three distinct sets of results in the literature. We take each set in turn.

\section{Motivation 1 - experimental observations}

The visualization studies by Kobayashi et al. (1983) and Kobayashi \& Izumi (1983) of rotating cones with slender half-angles show the existence of pairs of counter-rotating Görtler-type vortices prior to the appearance of turbulence. However, as the half-angle $\psi$ is increased beyond $40^{\circ}$, their visualizations clearly show that these vortices change to co-rotating vortices, as reported on rotating disks by Gregory et al. (1955), Kohama (1985), Reed \& Saric (1989), Kobayashi (1994), Corke \& Knasiak (1998), Saric et al. (2003), for example. Counter-rotating vortices are expected to arise from a dynamic instability induced by the centrifugal force of the flow field, and co-rotating vortices are attributed to an underlying crossflow instability. The experimental observations therefore strongly hint at alternative mechanisms over slender and broad cones.

\section{Motivation 2 - experimental measurements of the onset of turbulence}

Further evidence of distinct mechanisms is obtained by considering experimental measurements for the onset of turbulence by Kobayashi \& Izumi (1983) and Nickels \& Bertényi, University of Cambridge (personal communication, 2007) as compared to the onset of local absolute instability predicted by Garrett \& Peake (2007). Although the exact role of local absolute instability in transition over the rotating disk is less clear than originally proposed by Lingwood $(1995,1996)$ [see Davies \& Carpenter (2003), Pier (2003), Healey (2010), Imayama, Alfredsson \& Lingwood (2013)], the theoretical onset of local absolute instability is extremely close to numerous consistent measurements of the onset of turbulence over the rotating disk and this provides a useful of means of comparison. In particular, Garrett \& Peake demonstrated that the critical local Reynolds number for local absolute instability is independent of half-angle with $\mathrm{R}_{X} \approx 2.5 \times 10^{5}$; this can be compared to the two sets of experimental results:

i) Figure 1 shows the comparison with experimental measurements for the onset of turbulence reported by Kobayashi \& Izumi. For cones with $\psi \geq 60^{\circ}$, we see that transition occurs at a local Reynolds number independent of the half-angle and reasonably close to the predicted onset of local absolute instability. Note here that the precise definition of turbulent flow is somewhat subjective in experimental terms and Kobayashi \& Izumi's measurements are subject to some flexibility. This is clearly demonstrated by comparing their measurements for half-angles close to $90^{\circ}$ with Lingwood (1996)'s measurement on a rotating disk (the horizontal line at $90^{\circ}$ in Figure 1). This close agreement suggests that local absolute instability may well be involved in the transition over broad rotating cones, consistent with the rotating-disk flow. For more slender cones, the measured critical Reynolds numbers decrease sharply with decreased half-angle and occur in advance of the predicted onset of local absolute instability.

ii) Figure 2 shows Nickels \& Bertényi's measurements for the onset of turbulence over three cones (each with distinct half-angle) at different rotation rates. We see that the 


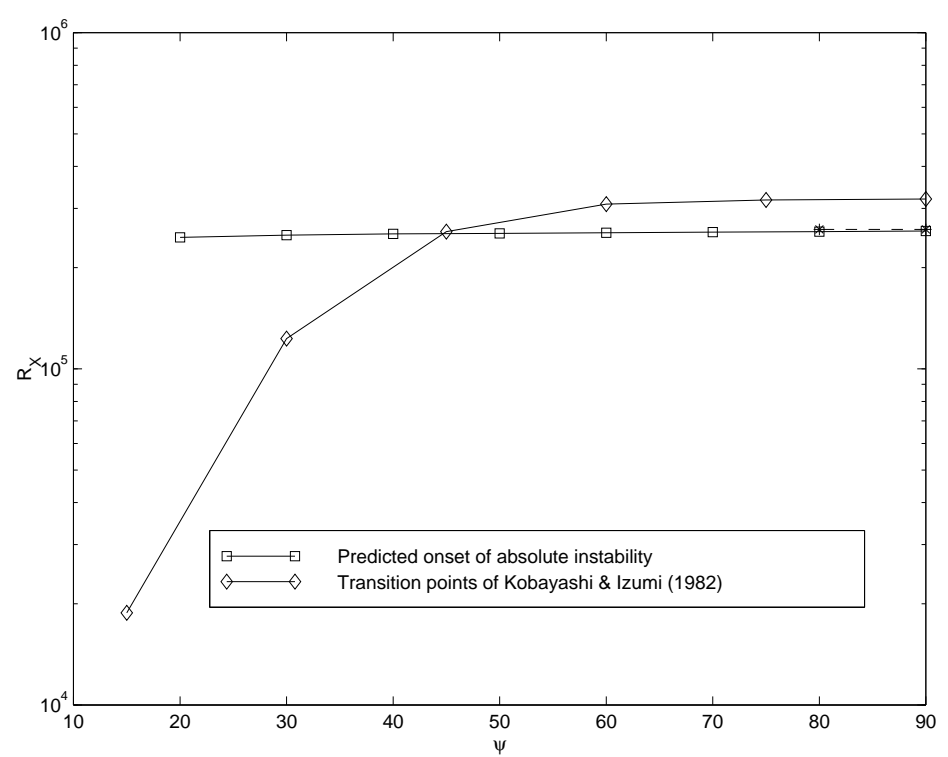

FiguRE 1. Critical $R_{X}$ for the onset of local absolute instability and the transitional values measured by Kobayashi \& Izumi (1983).

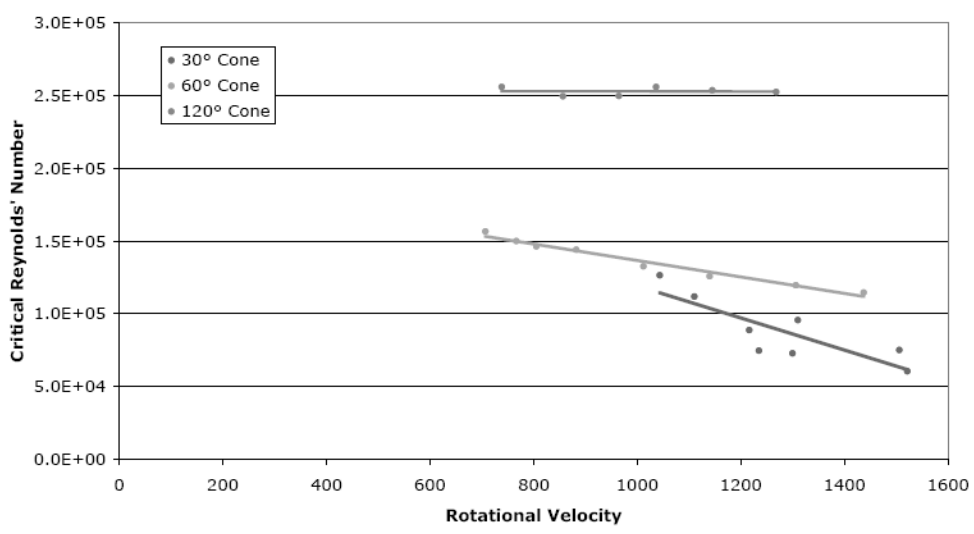

Figure 2. Experimental data due to Nickels \& Bertényi for the onset of turbulence, uppermost plot is $\psi=60^{\circ}$. (Cone angle $=2 \times \psi$ ).

measured critical Reynolds number over the broadest cone $\left(\psi=60^{\circ}\right)$ is in good agreement with the predicted onset of local absolute instability and is independent of rotation rate (which suggests that the dashed line in figure 1 can be extended to at least this halfangle). However, the onset of turbulence over the slender cones with $\psi=30^{\circ}$ and $15^{\circ}$ is again well in advance of the predicted onset of local absolute instability and dependent on the rotation rate. Furthermore, they reported greater variation in the flow behaviour through turbulent transition in the case of the most slender cone. This is thought to be linked to the greater prevalence of a secondary instability, which has been visualised in the form of horseshoe-like vortices that persist into the turbulent region of flow (see Kohama (1985)). 


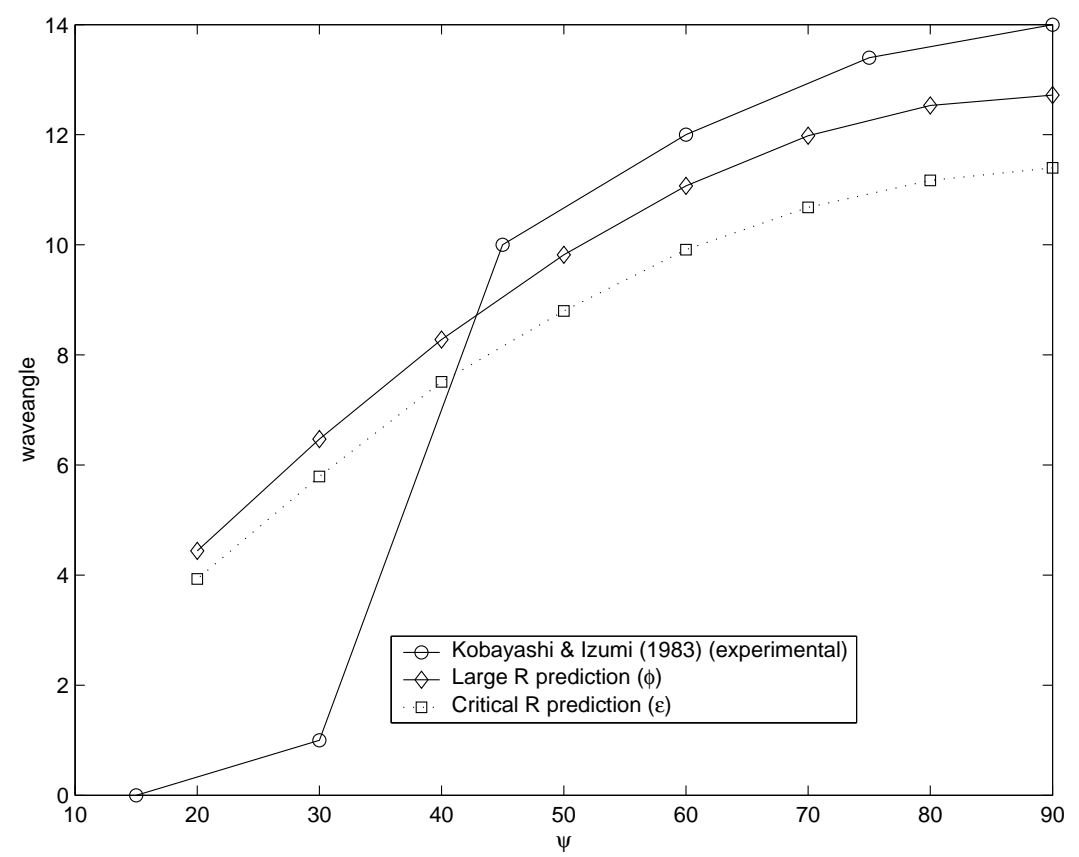

FiguRE 3. A comparison between the experimental observations of the vortex orientation angle and the predicted value at the onset of type I instability and the asymptotic limit for large Reynolds number. Garrett et al. (2009)

\section{Motivation 3 - predictions of convective instability}

Garrett et al. (2009) present mathematical studies of the rotating-cone boundary layers using a formulation consistent with other rotating-disk studies (Malik 1986 and Lingwood 1995, for example). They demonstrate that convective modes of type I and II (also known as crossflow and streamline-curvature modes, respectively) exist at all $10^{\circ} \leq \psi \leq 90^{\circ}$. The onset of convective instability is associated with the onset of the spiral vortices and the critical Reynolds numbers and other measurable quantities of the spiral vortices (number, angle of orientation) compare well with experimental observations by Kreith et al. (1962), Kappesser et al. (1973) and Kobayashi \& Izumi (1983) when $\psi \geq 40^{\circ}$. However, increasing discrepancy is found for $\psi<40^{\circ}$. This is clearly seen in Figure 3, for example, where the theoretical predictions of the orientation angle for vortices arising from the type I mode are compared to Kobayashi \& Izumi's experimental observations at each half-angle. Such comparisons suggest that the vortices found on slender cones cannot be attributed to the type I and II modes.

The behaviour of the type I and II modes was further elaborated by Garrett (2010) who extended the numerical results of Garrett et al. (2009) to consider the amplification rates of the modes through the convectively-unstable region as a function of half-angle. He found that the amplification rates of both mode types reduce with decreased halfangle. This finding is consistent with the hypothesis of a centrifugal mode that dominates at slender half-angles.

These three motivations give clear evidence for an instability mode arising from centrifugal effects that exists in addition to the well-known type I and II modes. We envisage all three modes existing within the flow over rotating cones of any half-angle, but with a relative dominance that depends on half-angle. It is therefore likely that a critical half- 
angle exists for the switch from a predominantly centrifugal instability (manifested in the appearance of counter-rotating vortices) to the crossflow instability (manifested in the appearance of co-rotating vortices) as the half-angle is increased. Although experiments have only been conducted at a small number of distinct half-angles, the evidence suggests this critical half-angle to be around $40^{\circ}$.

It is interesting to also note that for very slender cones $\left(\psi \leq 15^{\circ}\right)$, Kobayashi et al. (1983) and Kobayashi \& Izumi (1983) have observed both spiral and circular vortices. These are distinguished by non-zero and zero waveangles respectively, and have been observed for cones rotating in both otherwise still fluid and enforced axial flow. The theoretical study of these two cases is slightly different and we consider only spiral vortices in this paper. Our study of circular waves for $\psi \leq 15^{\circ}$ is presented as Hussain et al. (2012).

\section{Formulation}

We use coordinate axes aligned with the spiral vortices and with origin $O^{\prime}$ placed at the local position of the analysis. As shown in figure 4 , the $\hat{x}^{*}$-axis coincides with the direction of propagation of the spiral vortices aligned with the effective velocity direction. Alternatively, the $y^{*}$ - and $z^{*}$-axes are mutually orthogonal and run in the tangential and surface-normal directions, respectively (where a ${ }^{*}$ denotes a dimensional quantity in all that follows). The resulting coordinate system $\left(\hat{x}^{*}, y^{*}, z^{*}\right)$ rotates with the cone surface at constant angular frequency $\Omega^{\star}$. Importantly, the logarithmic spirals are directed such that the $y^{*}$-axis has a positive projection with respect to the direction of rotation of the cone. This requires that the $\hat{x}^{*}$-axis has positive projection onto the axis of rotation and the $y^{*}$-axis to have negative projection, as seen in Figure 4 . The spiral vortices are orientated at an angle $\phi$ relative to the circle formed from the planar cross-sectional normal to the axis of rotation of the cone. The governing dimensional Navier-Stokes equations are then derived in this co-ordinate system with appropriate scale factors.

This formulation is similar to that used in Kobayashi (1981) but differs by the sign of the Coriolis terms in the momentum equations, because we have used an anti-clockwise rotating cone, as opposed to a clockwise setup, for consistency when comparing results with our previous study for rotating cones with large $\psi$ (see Garrett et al. (2009)). Importantly, the a priori assumption that the spiral vortices exist and have orientation angle $\phi$ is a significant difference to our previous formulation in Garrett et al. (2009), where the existence and properties of vortices were an output of the analysis.

We non-dimensionalise lengths on a characteristic distance along the cone $l^{*}$, so that $\hat{x}^{*}=l^{*} \hat{x}$ and $y^{*}=l^{*} y$. Furthermore, we scale both logarithmic coordinates $\hat{x}$ and $y$, as well as the normal coordinate $z^{*}$, on the boundary-layer thickness, leading to the scaled coordinate system $(\check{x}, \bar{y}, \eta)=\mathrm{R}^{1 / 2}(\hat{x}, y, z)$ where $\mathrm{R}$ is the Reynolds number given by

$$
\mathrm{R}=\frac{\Omega^{*} l^{* 2} \sin \psi}{\nu^{*}}
$$

This scaling enables the vortex structure in both logarithmic directions to be analyzed at the same order as the length scale in the surface-normal direction. Usually a Görtler-mode analysis requires only lengths normal to the surface and spanwise to the vortices to be scaled on the boundary-layer thickness, see Hall (1982), Denier et al. (1991), however here the counter-rotating vortices are characterised by both logarithmic coordinates which require this scaling. This spatial scaling is another important difference to our previous formulation.

We assume that the spiral waves are periodic in the effective velocity direction and introduce periodicity into the perturbation quantities of vortex $\check{x}$-wavenumber $a$ and $\bar{y}$ - 


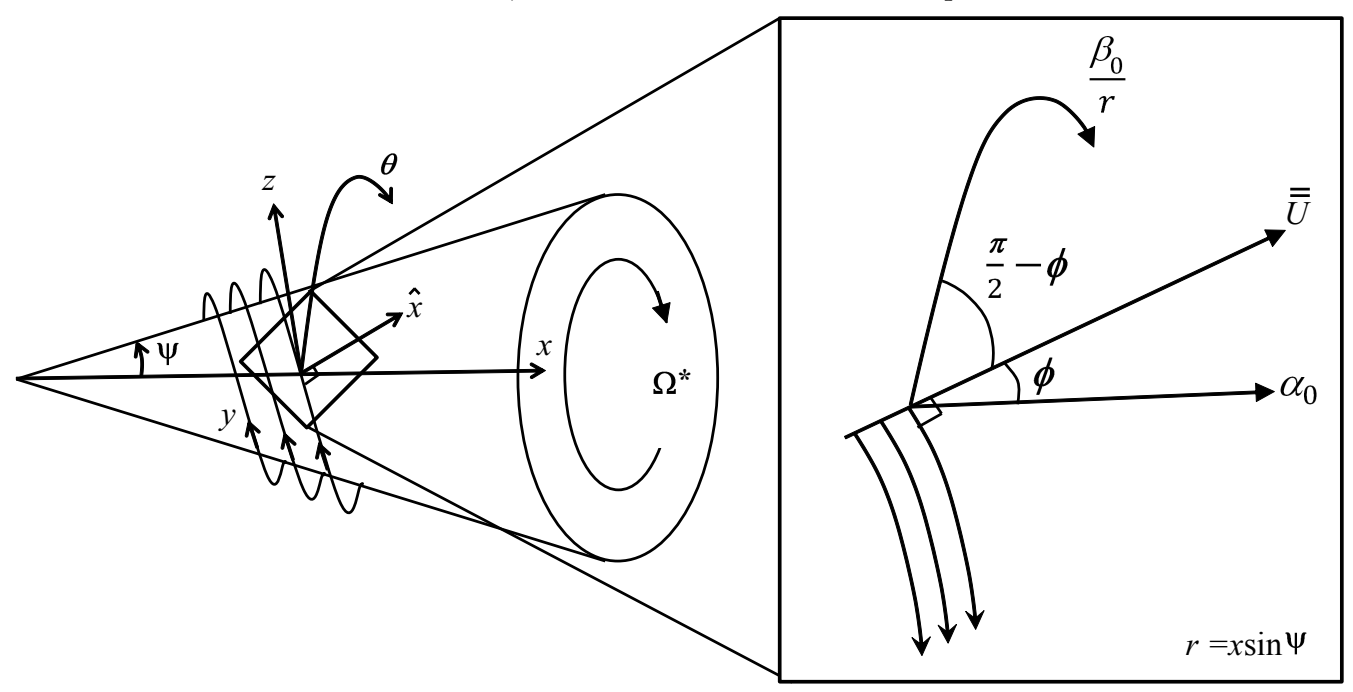

FIGURE 4. Diagram of spiral vortex instability of a rotating cone, showing conventional streamwise, aziumthal and surface-normal coordinates $(x, \theta, z)$ defined in Garrett et al. (2009), as well as the shifted logarithmic spiral coordinates $(\hat{x}, \theta)$ (left). Also included is a detailed physical interpretation (right) showing the streamwise, azimuthal and effective velocity directions $\overline{\bar{U}}$. Note that $\alpha_{0}$ and $\beta_{0} / r$ refer to wavenumbers in the streamwise and azimuthal directions of the previous formulation Garrett et al. (2009), whereas the logarithmic spiral coordinate $\hat{x}$ coincides with the effective velocity direction $\overline{\bar{U}}$.

wavenumber $b$. Scaling our perturbing quantities on the boundary-layer thickness, we introduce a perturbed flow of the form

$$
\tilde{\mathbf{u}}^{*}=\Omega^{*} l^{*} \sin \psi\left[\left\{x \tilde{U}(\eta ; \phi), x \tilde{V}(\eta ; \phi), \mathrm{R}^{-\frac{1}{2}} W\right\}+\mathrm{R}^{-\frac{1}{2}}\{\tilde{u}(\eta), \tilde{v}(\eta), \tilde{w}(\eta)\} \exp (\mathrm{i} a \check{x}+\mathrm{i} b \bar{y})\right] .
$$

where $x$ is streamwise direction over the cone, scaled on $l^{*}$ (see Garrett et al. (2009), for example). We note that these scalings remove $\psi$ from the governing equations. Similarly the pressure perturbation term scales as

$$
p^{*}=\left(\rho^{*} \Omega^{* 2} l^{* 2} \sin ^{2} \psi\right) \mathrm{R}^{-1} \tilde{p}(\eta) \exp (\mathrm{i} a \check{x}+\mathrm{i} b \bar{y}),
$$

where there is an associated corresponding base pressure, which is not stated here, as it is not required for the purposes of analysing the perturbation equations. The steady-flow components in the $\check{x}$ - and $\bar{y}$-directions are denoted $\tilde{U}(\eta ; \phi)$ and $\tilde{V}(\eta ; \phi)$ and can be found from the solution of the governing equations at leading order. Figure 5 gives these profiles for $\phi=0^{\circ}, 5^{\circ}$ and $10^{\circ}$ where we see a very slight increase in the velocity in the $\bar{y}$-direction as the waveangle is increased. However, we observe much more sensitivity for the velocity in $\check{x}$-direction, with a significant decrease in the far-field limit as the waveangle increases.

Importantly, $\tilde{U}$ and $\tilde{V}$ can be expressed as projections of the von Kármán profiles used in our previous formulation, for example in Garrett et al. (2009), as:

$$
\begin{aligned}
\tilde{U}(\eta ; \phi) & =U(\eta) \cos \phi+V(\eta) \sin \phi, \\
\tilde{V}(\eta ; \phi) & =U(\eta) \sin \phi+V(\eta) \cos \phi .
\end{aligned}
$$

This demonstrates consistency between this formulation and that used in previous studies. It is therefore clear that the velocity profiles for $\phi=0^{\circ}$ limit to those used previously, and in this formulation this corresponds to the circular waves observed for 
cones with $\psi \leq 15^{\circ}$. As discussed above, these are not considered here and the interested reader is referred to Hussain (2010), Hussain et al. (2012) for the associated stability analyses.

In order to derive the governing perturbation equations for spiral vortices $(\phi \neq 0)$, it is necessary to make a number of mathematical approximations to the scale factors which are based on the assumption of large Reynolds number and small waveangle. These can be justified from the experimental observations of Kobayashi \& Izumi which report $\phi \approx 0^{\circ}-2.7^{\circ}$. In $\S 4$ we investigate the short-wavelength asymptotic structure of the centrifugal instability and hence identify the spiral vortex wavenumber in the $\check{x}$-direction as $a=\epsilon^{-1}$, where $\epsilon$ is a small parameter. Here, $b=O(1)$ is the wavenumber in the $\bar{y}$-direction. Full details of the mathematical manipulations are given in Hussain (2010) and we arrive at the governing stability equations stated in Appendix A. We note that the governing equations are considerably more complicated than those considered under the previous formulation and this is a consequence of the different spatial scalings used here.

\section{Asymptotic analysis}

Analytical progress is made for large vortex wavenumbers, which form the basis of our asymptotic structure. This is in contrast to Garrett et al. (2009), for example, who present asymptotic analyses of type I and II modes by introducing a small parameter given by inverse powers of the Reynolds number as the basis of the asymptotic structure. Their analysis leads to explicit expressions for the wavenumbers of neutrally-stable modes that enable simple comparisons with the upper and lower branches of the numericallycomputed neutral curves in the large Reynolds-number limit, as can be seen in Figures 10 \& 11 of Garrett et al. (2009). In this study, however, the governing equations are solved to determine leading- and next-order estimates of a scaled Taylor number for neutrallystable modes. Comparisons with other results expressed in terms of Reynolds numbers are therefore less immediate and this is further discussed in $\S 6$. The asymptotic approach presented here is clearly distinct to that used previously and follows Hall (1982) for the Taylor problem of flow between concentric rotating cylinders. Indeed, for slender rotating cones, $\psi$ is sufficiently small that the formulation resembles that for flow moving axially over a rotating cylinder.

The Taylor number in this formulation is given by

$$
T=\frac{2 \cot \psi \cos \phi}{\sin ^{5} \psi}
$$

We consider it to be a function of $\psi$ and parameterised by the particular $\phi$ under consideration. The expression is such that $T$ increases with decreased $\psi$ and can be considered as a measure of cone slenderness for particular $\phi$. The Taylor number can be thought of as characterizing the importance of centrifugal forces relative to viscous forces, and is closely related to the Görtler number, which has been used to describe centrifugal instabilities, for example in fully developed and boundary layer flows by Hall (1982).

\section{Leading-order solution}

The perturbation quantities are expanded and we consider a WKB solution for small values of $\epsilon$. Recall that $a=\epsilon^{-1}$ with $a$ the wavenumber in the $\check{x}$-direction. The dominant terms in the governing equations (A 1)-(A 4) balance if we scale $T \sim \epsilon^{-4}$ and $W / V \sim$ 

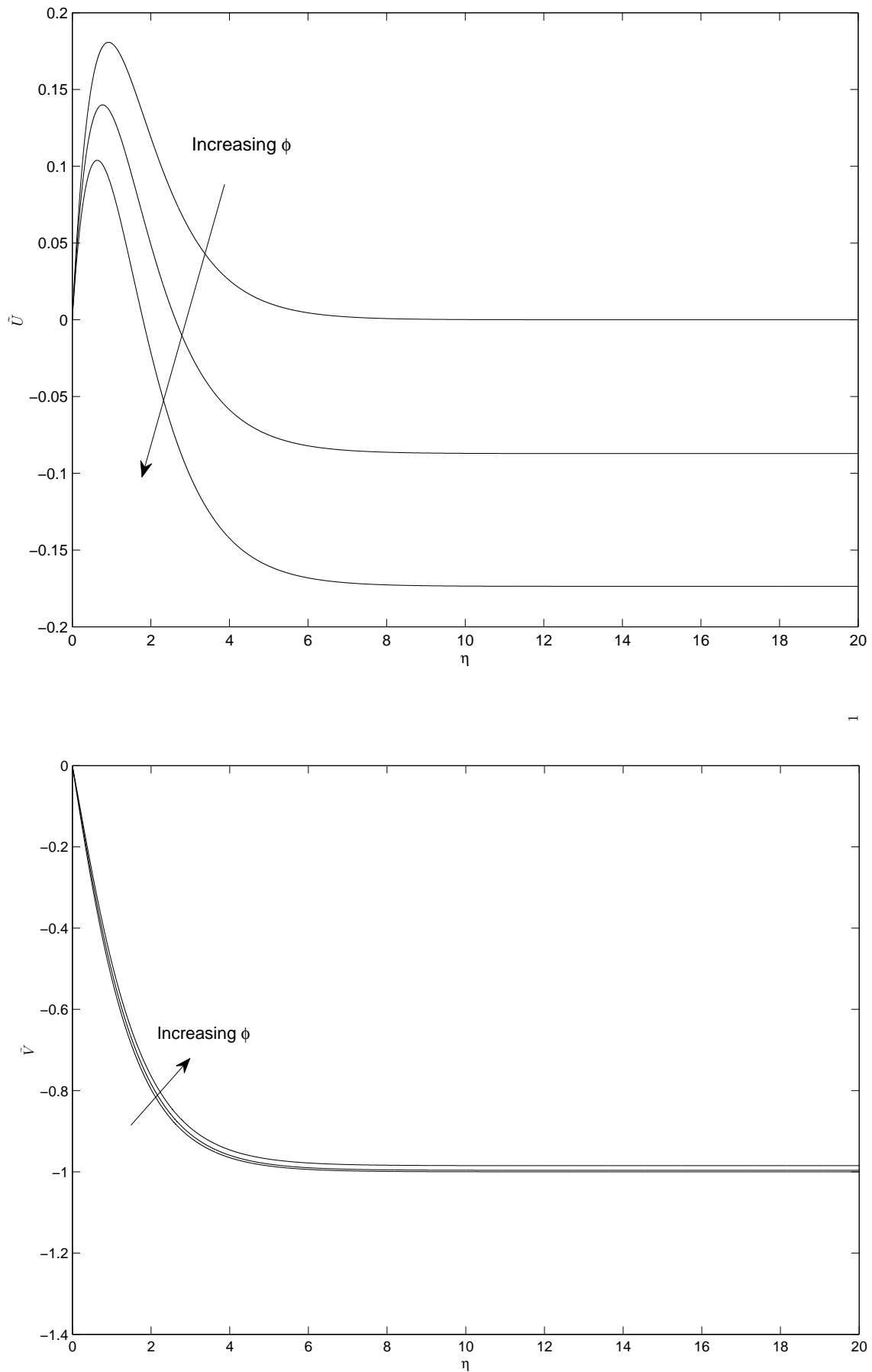

Figure 5. Effective velocity profiles $\tilde{U}$ and $\tilde{V}$ for waveangles $\phi=0^{\circ}, 5^{\circ}$ and $10^{\circ}$ (in direction of arrow). 
$O\left(\epsilon^{-2}\right)$, resulting in

$$
\begin{aligned}
\tilde{u} & =E\left(u_{0}(\eta)+\epsilon u_{1}(\eta)+\epsilon^{2} u_{2}(\eta)+\ldots\right), \\
\tilde{v} & =\epsilon^{2} E\left(v_{0}(\eta)+\epsilon v_{1}(\eta)+\epsilon^{2} v_{2}(\eta)+\ldots\right), \\
\tilde{w} & =E\left(w_{0}(\eta)+\epsilon w_{1}(\eta)+\epsilon^{2} w_{2}(\eta)+\ldots\right), \\
T & =\epsilon^{-4}\left(\lambda_{0}+\lambda_{1} \epsilon+\lambda_{2} \epsilon^{2}+\ldots\right),
\end{aligned}
$$

where $\lambda=\lambda_{0}+\lambda_{1} \epsilon+\lambda_{2} \epsilon^{2}+\ldots, E=\exp \frac{\mathrm{i}}{\epsilon} \int^{\varphi} K(\tau) \mathrm{d} \tau$ and $\varphi=\frac{\sin \psi}{\bar{h}_{1}} \eta$. Importantly, we note that the definition of the Taylor number (4.1) arises following differentiation with respect to $\eta$ and consequent manipulation of the governing disturbance equations (A 1)(A 4), following the method of Hall (1982) to identify the correct scaling for the Görtler number, and consequently the Taylor number. After substitution of these expansions into the governing equations and some simplification owing to the assumption of small waveangle, we arrive at an eigenrelation at leading order which can be solved to give the scaled leading-order eigenvalue estimate

$$
\bar{\lambda}_{0}=-\frac{1}{\left[(\tilde{V} \cos \phi+1) \frac{\partial \tilde{V}}{\partial \eta}\right]_{\min }},
$$

where the scaled eigenvalue is $\bar{\lambda}=\lambda \bar{h}_{1}^{4}(1+\check{x} \cos \phi-\bar{y} \sin \phi)$ and $\bar{h}_{1}$ is a scale factor defined as $\bar{h}_{1}=1+\check{x} \cos \phi-\bar{y} \sin \phi+\eta \cos \psi \sin ^{2} \phi$. In the range of small waveangles that have been experimentally observed for slender cones, the minimum value of the denominator occurs at the wall $(\eta=0)$. In order to evaluate the denominator, we note that $\tilde{V}(0)=0$ and evaluate only $\left|\tilde{V}^{\prime}(0)\right|$ for varying $\phi$. Although not shown here, this quantity is found to decrease as $\phi$ is increased and our leading-order eigenvalue estimate increases in magnitude as the waveangle is increases. Numerical values for $\bar{\lambda}_{0}$ are given in Table 1 for various $\psi$ and $\phi$ relevant to experimental observations.

\section{First-order solution}

Following Hall's method, we seek to analyze vortex activity, which from the leading order solution is found to be located at the wall near $\eta=0$, through obtaining the location of the minimum of the function $(\tilde{V} \cos \phi+1) \frac{\partial \tilde{V}}{\partial \eta}$. Hence, we consider a thin layer about this location, which is of thickness $\mathrm{O}\left(\epsilon^{\frac{2}{3}}\right)$, and expand the Taylor number in the form

$$
T=\epsilon^{-4}\left(\lambda_{0}+\lambda_{1} \epsilon^{\frac{2}{3}}+\ldots\right) .
$$

Furthermore, we re-scale the normal variable on an appropriate thickness $\xi=\frac{\varphi}{3^{\frac{1}{3}} \epsilon^{\frac{2}{3}}}$. The normal perturbation velocity is then expanded as

$$
\tilde{w}=w_{0}(\xi)+\epsilon^{\frac{2}{3}} w_{1}(\xi)+\ldots,
$$

with $\tilde{u}=O(1)$ and $\tilde{v}=O\left(\epsilon^{2}\right)$ as in the leading-order analysis. After substituting these expressions into the governing equations and equating terms of $O\left(\epsilon^{\frac{2}{3}}\right)$ we obtain an eigenvalue relation at first order. This can be solved to give a first-order estimate of our scaled Taylor-number eigenvalue as

$$
\bar{\lambda}_{1}=\frac{2.3381 \times 3^{\frac{1}{3}}}{\left|\tilde{V}^{\prime}(0)\right|}\left[\frac{\tilde{V}^{\prime \prime}(0)}{\tilde{V}^{\prime}(0)}+\tilde{V}^{\prime}(0) \cos \phi\right]^{2} .
$$

The mathematics is very involved and full details are given by Hussain (2010). In fact we obtain an infinite sequence of eigenvalues $\left\{\lambda_{1 n}\right\}$, corresponding to the zeros of an Airy 


\begin{tabular}{c|ccc}
$\psi$ & $\phi$ & $\bar{\lambda}_{0}$ & $\bar{\lambda}_{1}$ \\
\hline $15^{\circ}$ & $0^{\circ}$ & 1.6236 & 2.0769 \\
\hline $30^{\circ}$ & $1^{\circ}$ & 1.6477 & 1.8567 \\
& $2^{\circ}$ & 1.6731 & 1.6389 \\
& $2.7^{\circ}$ & 1.6915 & 1.4887 \\
& $4^{\circ}$ & 1.7277 & 1.2162 \\
& $5^{\circ}$ & 1.7572 & 1.0146 \\
& $6^{\circ}$ & 1.7883 & 0.8221 \\
& $8^{\circ}$ & 1.8556 & 0.4747 \\
\hline \multirow{2}{*}{$45^{\circ}$} & $8.5^{\circ}$ & 1.8735 & 0.3979 \\
& $10^{\circ}$ & 1.9305 & 0.1991
\end{tabular}

TABLE 1. Leading- and first-order eigenvalue estimates of the scaled Taylor number for orientation angles as observed by Kobayashi \& Izumi on cones with the stated half-angle.

function on the negative real axis. Numerical values for the most dangerous $\bar{\lambda}_{1}$ are given in Table 1 for various $\psi$ and $\phi$ relevant to experimental observations.

Asymptotic estimate of Taylor number

Combining the leading- and next-order solutions, the most dangerous instability mode has a scaled Taylor-number expansion given by

$$
\begin{aligned}
\bar{T} & =T \bar{h}_{1}^{4}(1+\check{x} \cos \phi-\bar{y} \sin \phi) \\
& =\epsilon^{-4}\left(\frac{1}{\left|\tilde{V}^{\prime}(0)\right|}+\frac{2.3381 \times 3^{\frac{1}{3}} \epsilon^{\frac{2}{3}}}{\left|\tilde{V}^{\prime}(0)\right|}\left[\frac{\tilde{V}^{\prime \prime}(0)}{\tilde{V}^{\prime}(0)}+\tilde{V}^{\prime}(0) \cos \phi\right]^{2}+\ldots\right) .
\end{aligned}
$$

Numerical estimates of the leading- and first-order eigenvalues corresponding to the scaled Taylor number are shown in Table 1 for parameter values in the range of those experimentally observed by Kobayashi \& Izumi (1983).

Plots of the scaled Taylor number against wavenumber, $\epsilon^{-1}=a$, for $\phi=0^{\circ}-10^{\circ}$ in increments of $2.5^{\circ}$ are shown in Figure 6. The unstable region is above the curves and the stable region below. We see that there is slight variation with waveangle that reduces with increased wavenumber. At this stage it is sufficient to take that Equation (4.2) demonstrates the existence of the centrifugal mode; the results will be interpreted in $\S 6$.

\section{Numerical analysis}

We now discuss the numerical solution of the system described in $\S 3$. The formulation is reasonably consistent with that presented above, however rather than solving (A 1)-(A 4) using expansions in $\epsilon$, we make further approximations and manipulations to convert these equations into the governing fourth-order Orr-Sommerfeld (OS) equation for stationary disturbances within the system. To this end, we begin by neglecting terms arising from Coriolis and streamline-curvature effects and collate the resulting equations 


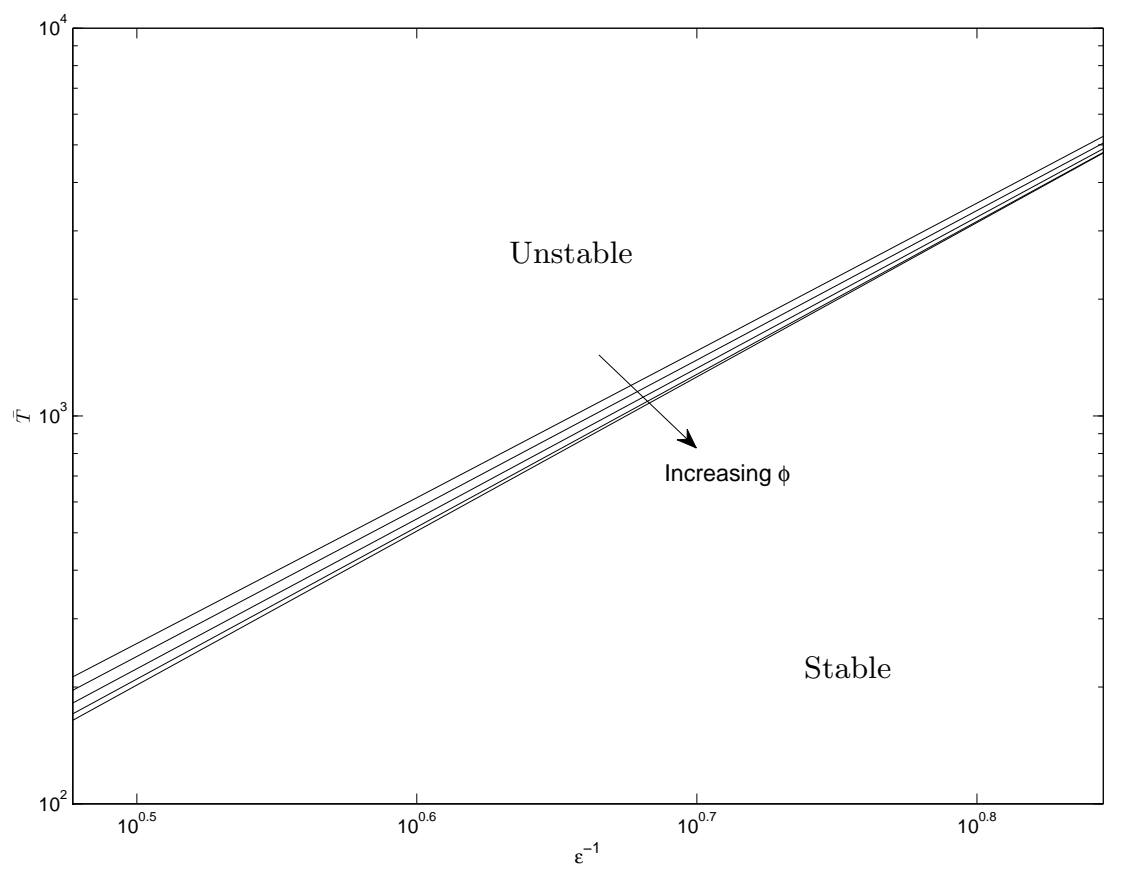

FiguRE 6. Asymptotic scaled Taylor number $\bar{T}$ as a function of non-dimensional vortex wavenumber $\epsilon^{-1}$ for $\phi=0^{\circ}-10^{\circ}$ in increments of $2.5^{\circ}$. The unstable region is above the neutral curves.

in terms of the normal perturbation velocity to form

$$
\left[\mathrm{i}\left(\frac{\partial^{2}}{\partial \eta^{2}}-k^{2}\right)^{2}+\operatorname{Re}\left(\alpha_{1} \tilde{U}+\beta_{1} \tilde{V}\right)\left(\frac{\partial^{2}}{\partial \eta^{2}}-k^{2}\right)-\operatorname{Re}\left(\alpha_{1} \frac{\partial^{2} \tilde{U}}{\partial \eta^{2}}+\beta_{1} \frac{\partial^{2} \tilde{V}}{\partial \eta^{2}}\right)\right] \tilde{w}=0
$$

where

$$
\alpha_{1}=\frac{a \sin \psi}{R e}, \quad \beta_{1}=b \sin \psi, \quad k=\sqrt{\alpha_{1}^{2}+\beta_{1}^{2}},
$$

and $R e=x \sin \psi$ is the local Reynolds number, interpreted as the local non-dimensional radius of the cone surface from the axis of rotation. We can relate this rotational Reynolds number, $R e$, to the conventional Reynolds number, $\mathrm{R}$, defined in $\S 3$ using equation (8) of Kobayashi \& Izumi (1983) to re-write the surface-curvature term, which leads to the relation

$$
R e=\mathrm{R}^{\frac{1}{2}} \sqrt{1.616} .
$$

We note here that the dependence of the system on the vortex waveangle, $\phi$, is purely in the form of the projected basic flow velocities $\tilde{U}(\eta ; \phi), \tilde{V}(\eta ; \phi)$. Essentially, the vortex waveangle forms an input to the problem, which allows the OS equation above to be solved whilst using various projected basic flows as input functions. We proceed to investigate the solutions for $\psi=15^{\circ}, 30^{\circ}$ and $45^{\circ}$, noting that $\phi=0^{\circ}, 2.7^{\circ}$ and $8.5^{\circ}$, respectively, thereby yielding results in a suitable parameter regime, which can be compared with the findings of Kobayashi \& Izumi (1983).

In order to solve the governing fourth-order perturbation equation (5.1), we employ an 


\begin{tabular}{c|ccc}
$\psi$ & $\phi$ & $R e_{c}$ & $\alpha_{1, c}$ \\
\hline $15^{\circ}$ & $0^{\circ}$ & 50 & 1.01 \\
$30^{\circ}$ & $2.7^{\circ}$ & 135 & 0.72 \\
$45^{\circ}$ & $8.5^{\circ}$ & 270 & 0.59
\end{tabular}

TABLE 2. Numerical calculations of the critical Reynolds numbers, $R e_{c}$, and critical vortex wavenumbers, $\alpha_{1, c}$, in the effective velocity direction for a range of small half-angle cones and corresponding vortex waveangles.

existing OS solver routine for the rotating cone, which has been modified to allow existing solutions for the OS neutral curve at specific values of $\psi$ to be used in order to enable fast convergence when searching for neutral curves for the required values of $\psi$ for slender cones. Specifically, this routine has been coded to enable the upper branch neutral curve solutions for larger half-angles (eg. $\psi=50-70^{\circ}$ ) to be used as input solutions when solving the OS equation (5.1) for smaller half-angles (such as $\psi=45^{\circ}$ ). Provided the corresponding base flows for each specific half-angle are input into the OS solver, neutral curve solutions at the larger half-angle are used to obtain the OS solution for the smaller half-angle. Hence, we are able to arrive at convergent neutral curves, gradually merging from previously obtained larger half-angles (such as $\psi=45^{\circ}$ ), and moving towards much slender cones of half-angle $\left(\psi=15^{\circ}\right)$. We aim to obtain neutral stability curves for the required values of $\psi=15^{\circ}, 30^{\circ}$ and $45^{\circ}$, using the corresponding vortex waveangle values $\phi=0^{\circ}, 2.7^{\circ}$ and $8.5^{\circ}$, respectively.

Numerical predictions of the critical Reynolds numbers and critical vortex wavenumbers for $\psi=15^{\circ}, 30^{\circ}$ and $45^{\circ}$ are shown in table 2 . The results illustrate a decrease in the half-angle leads to a reduction in the critical Reynolds number, implying slender rotating cones represent the most unstable flow cases and cones of $\psi \leq 15^{\circ}$ harbour the most dangerous modes. This is corroborated by the critical vortex wavenumbers in the effective velocity direction, which increase for smaller values of $\psi$. In the case of steady flow, this would lead to greater amplification rates. Furthermore, while the results recover the findings of Kobayashi \& Izumi (1983), more importantly, at large Reynolds numbers and large vortex wavenumbers, we observe reasonably good qualitative agreement with our asymptotic results, as discussed below in $\S 6$.

It is useful to note at this stage, to enable suitable comparison with the asymptotic analysis of $\S 4$, we are interested in the vortex wavenumber in the effective velocity $\check{x}$ direction, namely $\alpha_{1}$. We recall that the wavenumber in the $\bar{y}$-direction, $b=O(1)$, as outlined in Hussain (2010).

\section{Comparison between asymptotic and numerical analysis}

In this section, we present comparisons between the large vortex wavenumber asymptotics in $\S 4$ and numerical OS neutral curve predictions in $\S 5$. In the case of the latter, previous studies for the rotating disk (for example Lingwood (1995) and Malik (1986)) have shown that the relative importance of the type II stability mode is unable to be predicted. However, analysis of the OS equation does yield qualitatively correct predictions of the neutral stability curves, which increase in numerical accuracy as $R e \rightarrow \infty$ (see, for example, Garrett et al. (2010) for broad rotating cones). Hence, the results of the OS analysis presented should compare well in theory with the large vortex wavenumber asymptotics at high Reynolds number. 


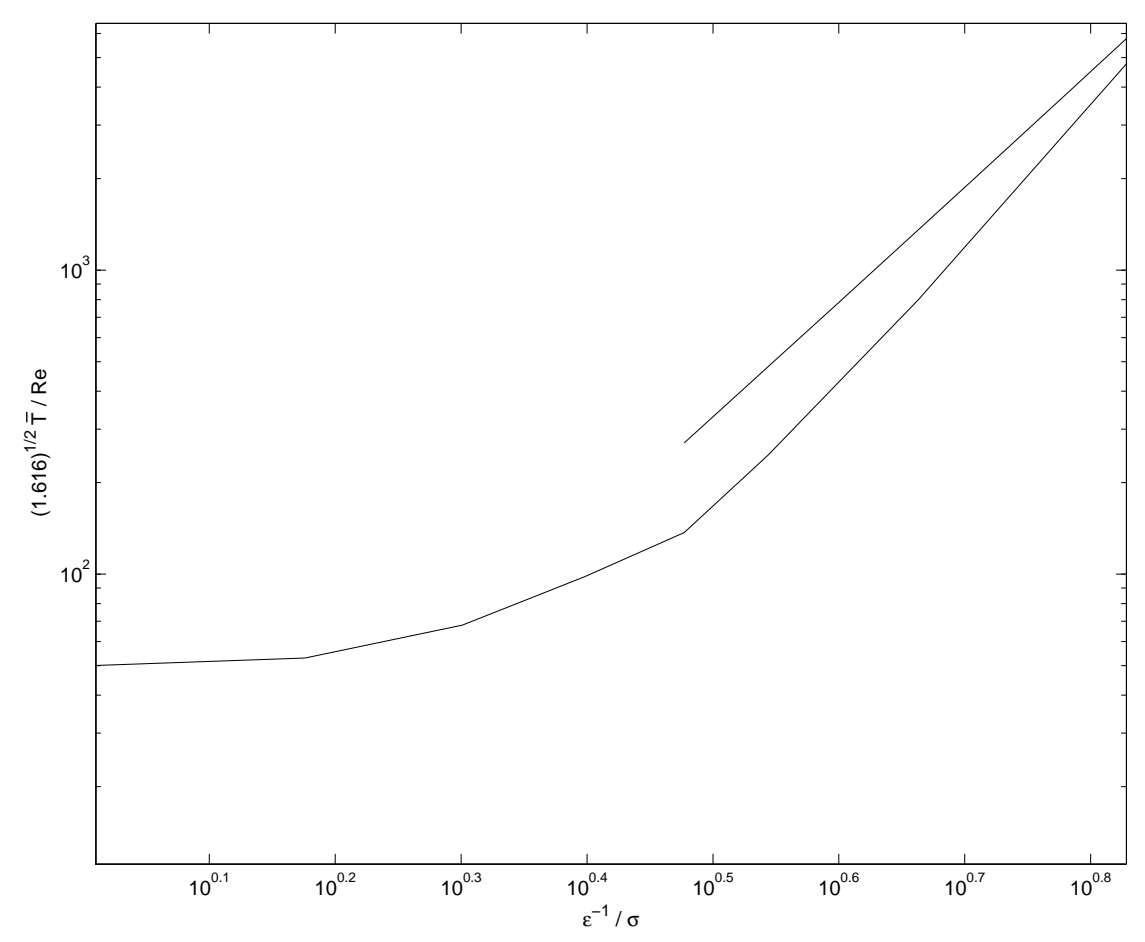

Figure 7. A comparison between the scaled effective asymptotic Taylor number $\bar{T}$ (above) and the Reynolds number Re predicted by the OS analysis (below), against vortex wavenumbers $\epsilon^{-1}$ and $\sigma$ respectively, for $\psi=15^{\circ}, \phi=0^{\circ}$.

Appropriate scalings are used, following Hussain (2010) so that the Taylor number defined in equation (4.1) is linearly related to the rotational Reynolds number (see also Kobayashi \& Izumi (1983)), which leads to a relationship of the form

$$
R e=\bar{T} \sqrt{1.616}
$$

for larger Reynolds number, Re, and large Taylor number, $T$. The definition of the Taylor number is such that increased $\bar{T}$ corresponds to reduced half-angle (for any fixed waveangle). The results may therefore suggest that the most unstable case (in the asymptotic sense of a broader range of unstable wavenumbers) for the centrifugal-instability mode is for small $\psi$ and $\phi=0^{\circ}$; this, however, assumes a particular behaviour for any upper neutrally-stable branch in that it is at worst parallel to the computed lower branch. Care also should be taken in the interpretation of these results owing to the scale factors introduced.

In the case of cones with half-angles $\psi \leq 15^{\circ}$, Kobayashi \& Izumi's results for $\psi=15^{\circ}$ are recovered for large Reynolds number and large vortex wavenumber $\epsilon^{-1}$ in Figure 9.2 of Hussain (2010). Furthermore, discussion of the formulation, analysis and results in this parameter regime where circular vortices are observed is given in Hussain et al. (2012).

Comparisons are sought for large vortex wavenumber, $\epsilon^{-1}$, between the scaled effective asymptotic Taylor number, $\bar{T}$, and the numerical OS neutral curves depicting Reynolds number, $R e$, in terms of the vortex wavenumber $\sigma$ in the effective velocity direction. We note from equation (3) in Kobayashi \& Izumi (1983) that this vortex wavenumber is 


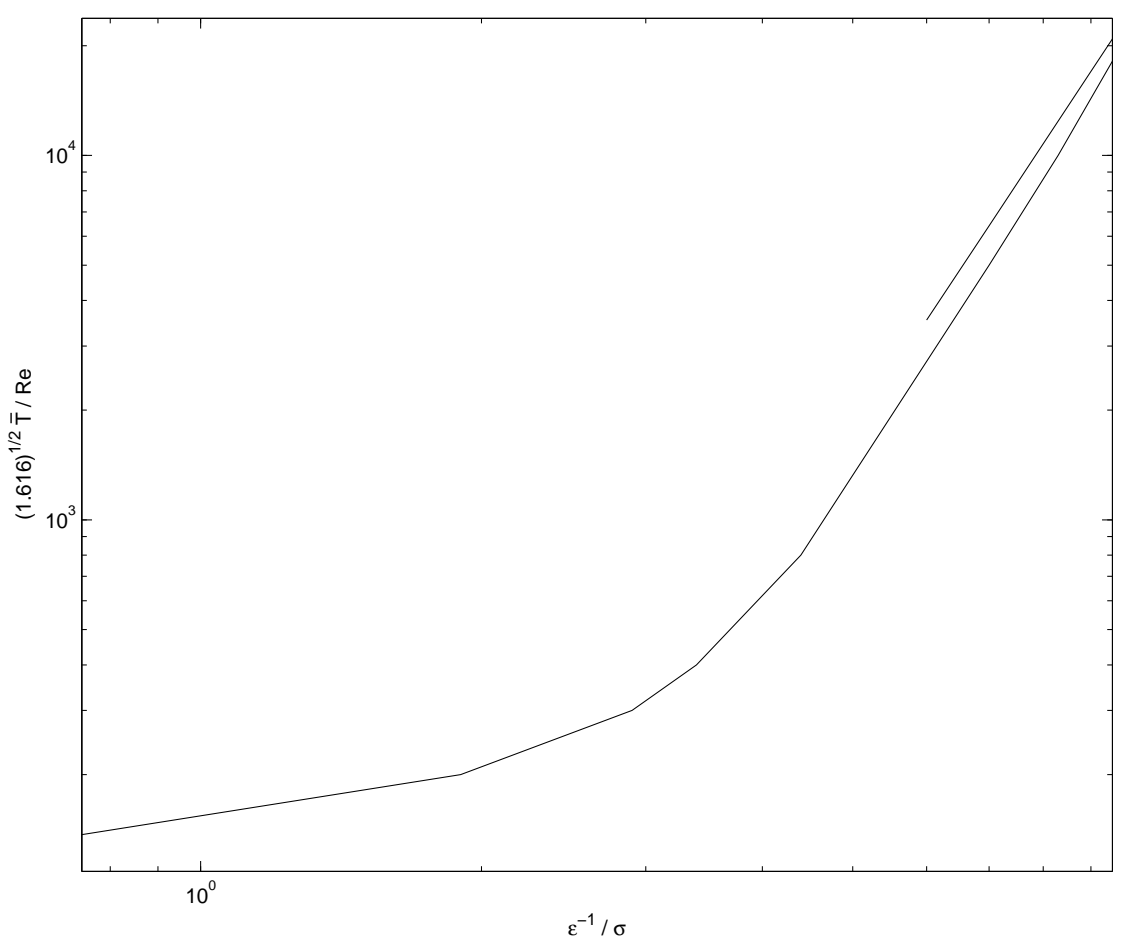

FiguRe 8. A comparison between the scaled effective asymptotic Taylor number $\bar{T}$ (above) and the Reynolds number $R e$ predicted by the OS analysis (below), against vortex wavenumbers $\epsilon^{-1}$ and $\sigma$ respectively, for $\psi=30^{\circ}, \phi=2.7^{\circ}$.

expressed as $\sigma=\alpha \delta_{1} \sin \theta$, where $\theta$ represents the cone half-angle, $\psi$, in the current study. Furthermore, $\alpha$ is linearly related to $a$ by a factor of $\sin \psi$, such that $a=\alpha \sin \psi$. After some manipulations of the definitions used in Kobayashi \& Izumi (1983) and comparing the OS equation (5.1) to that presented in equation (4) of Kobayashi \& Izumi (1983), we note that $\sigma$ used in Kobayashi \& Izumi (1983) is analogous to $\alpha_{1}$ in the current numerical analysis, representing the vortex wavenumber in the effective velocity direction. Importantly, we note due to the fact that $b=O(1)$, the stability characteristics of the problem depend mainly on the vortex wavenumber in the effective velocity direction, $a$. Physically, this is consistent with figure 4 , as there is a large number of vortex spirals, which are periodic along the effective velocity direction, indicating boundary layer growth along this axis, but not normal to this in the $\bar{y}$-direction. This is also observed in a number of experimental studies, including Kobayashi \& Izumi (1983), and is the reason why a short-wavelength asymptotic analysis is applicable in $\S 4$.

Using the appropriate form for the scaled effective asymptotic Taylor number, $\bar{T}$, expanded in terms of vortex wavenumber $\epsilon^{-1}$, we present comparisons with the Reynolds number $R e$ predicted by the OS analysis against $\sigma$ in figures 7,8 and 9 for $\psi=15^{\circ}, 30^{\circ}$ and $45^{\circ}$, respectively. In each figure, we have computed asymptotic curves for $\phi=0^{\circ}, 2.7^{\circ}$ and $8.5^{\circ}$, respectively, in order to identify and compare with the most suitable basic flow used within the OS analysis. These values correspond to the theoretical values of $\phi$ 


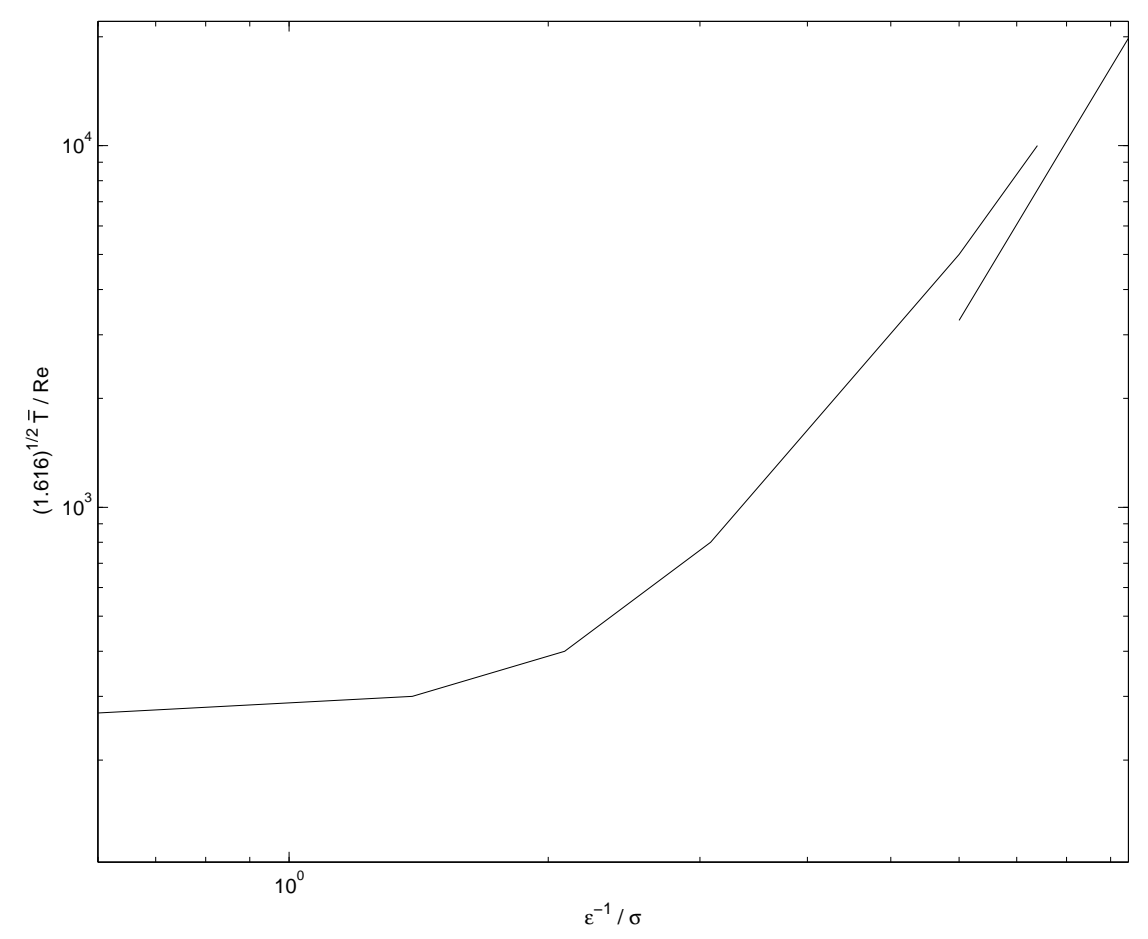

Figure 9. A comparison between the scaled effective asymptotic Taylor number $\bar{T}$ (below) and the Reynolds number Re predicted by the OS analysis (above), against vortex wavenumbers $\epsilon^{-1}$ and $\sigma$ respectively, for $\psi=45^{\circ}, \phi=8.5^{\circ}$.

presented in Kobayashi \& Izumi (1983) and hence facilitate a useful comparison with their results, as shown in Hussain et al. (2012) for $\psi=15^{\circ}$ specifically.

We observe relatively good qualitative agreement between the OS neutral curve measurements and the asymptotic branches of the scaled effective Taylor number for all three cases $\psi=15^{\circ}, 30^{\circ}$ and $45^{\circ}$. However, in particular, we notice that as $\psi$ increases, the agreement between the asymptotics and numerics becomes more favourable for larger values of $\bar{T}$, further along the asymptotic branch, which can be seen as we move from figures 7 to 9 .

One explanation is clearly, as the asymptotic analysis is developed for large vortex wavenumbers and large Reynolds numbers, its comparison with the numerical OS curves should be closer as we move further along the upper branch, for large Re and large $\sigma$.

However, another explanation for the stronger agreement for lower values of $\psi$ may arise from the fact the numerical analysis is built on the appropriate length scalings governing a centrifugal instability based on $\psi<45^{\circ}$. These numerical OS measurements should increase in accuracy as $\psi$ is reduced. Hence, the comparisons for increased values of $\psi$ close to $45^{\circ}$ should become more favourable at larger Reynolds numbers and larger vortex wavenumbers. This is also a property of the numerical OS analysis in that predictions increase in numerical accuracy for larger Reynolds numbers, as $R e \rightarrow \infty$.

More importantly, as mentioned in Kobayashi \& Izumi (1983), there appears to be a continuous change from counter-rotating vortices to unidirectional (co-rotating), as the cone half-angle increases from $\psi=15^{\circ}$. This was predicted in Garrett et al. (2009) to 


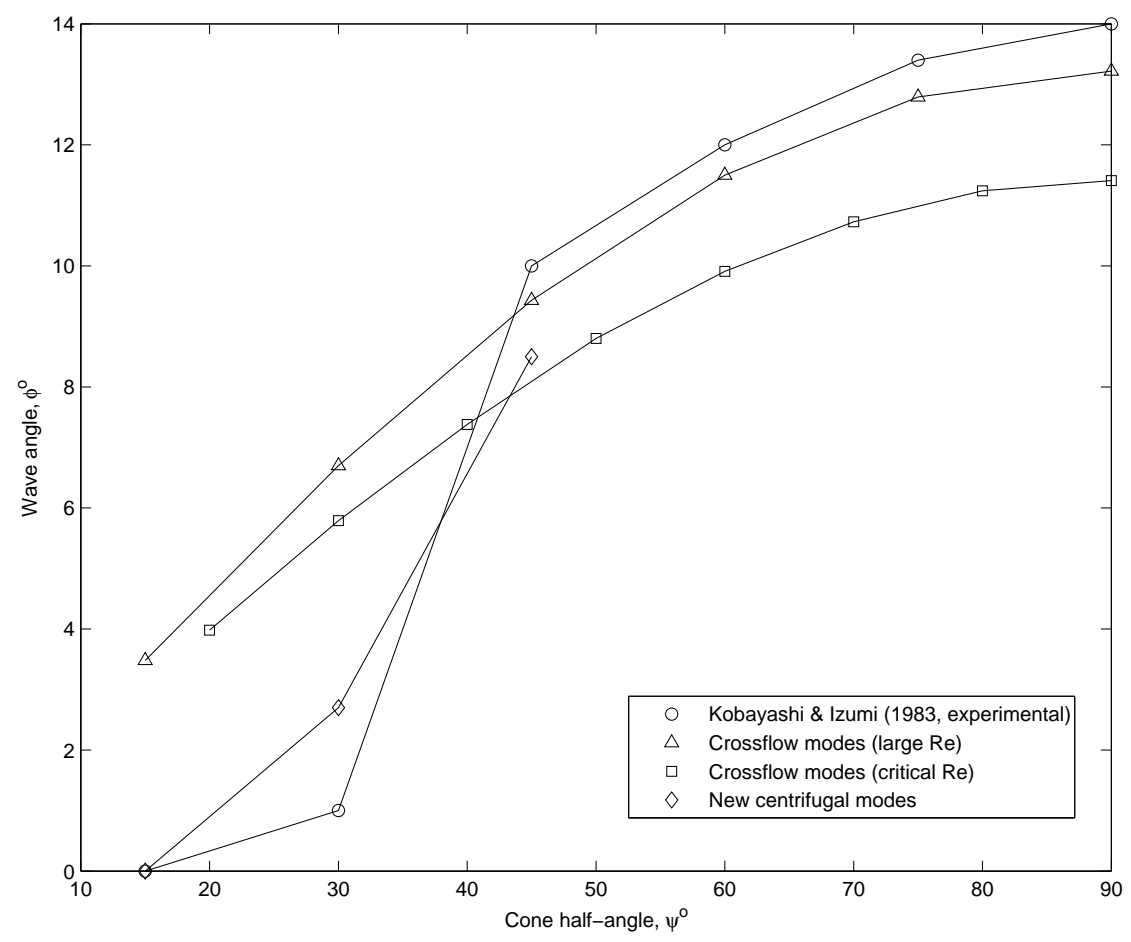

FiguRE 10. A comparison between experimental observations and theoretical predictions of the vortex orientation angle at the onset of instability, updated from Garrett et al. (2009). The diagram illustrates the competing nature of the type I (crossflow) instability versus the new (centrifugal) instability modes, which dominate for slender half-angles and compare well with the experimental measurements in this regime.

be caused by a gradual change in the dominant underlying instability mechanism from one of crossflow instability (for large $\psi$ ) to a centrifugal instability (for small $\psi$ ). Therefore, the numerical OS analysis, which is based on scalings consistent with a centrifugal instability, should attain more accurate predictions for smaller half-angles. However, the measurements would require larger Reynolds numbers and larger vortex wavenumbers to yield similarly favourable comparisons for increased cone half-angles, which is the case observed in figures 8 and 9 , when compared with figure 7 .

\section{Conclusion}

In this paper we have highlighted the motivation for the hypothesis of a centrifugalinstability mode within the general class of rotating-cone boundary-layer flows. An alternative formulation that focuses on centrifugal effects has been developed and independent asymptotic and numerical analyses conducted to verify the existence of such a mode.

Although the asymptotic analysis was used initially to identify the centrifugal mode, it yields an indication of the range of unstable wavenumbers against half-angle only for large Reynolds numbers and large vortex wavenumbers. In this regime, the range of stable wavenumbers increases with reduced half-angle (which is, in the context of the 
centrifugal instability, stabilizing). Nevertheless, the asymptotic approach is unable to calculate critical Reynolds numbers and amplification rates.

However, the OS numerical analysis presented confirms existence of the centrifugal mode and reveals a reduction in the critical Reynolds number as well as an increase in the amplification rate with reduced half-angle, suggesting smaller values of $\psi$ are actually destabilizing. Hence, for flow over a spinning cone surface with a relatively small half-angle, the centrifugal-instability mode may be interpreted physically as the most dangerous.

We conclude that three instability modes exist within the boundary-layer flow over rotating cones: crossflow (type I) and streamline curvature (type II), which have been observed previously in Garrett et al. (2009) to dominate for larger values of $\psi>40^{\circ}$; and a distinct centrifugal mode presented in this study, which appears to dominate for small $\psi<40^{\circ}$. Importantly, this mode differs from the streamline curvature (type II) mode for larger half-angles, which arises due to viscous effects close to the cone surface. Meanwhile, the centrifugal mode for small half-angles arises from the centrifugal forces present in the mean flow for small $\psi$, due to the effects of curvature of the cone surface. Consequently, this mode is visualised as helical spiral vortices of a counter-rotating nature, which wrap around the cone surface.

To this end, figure 10 shows an updated view of the results initially presented in Garrett et al. (2009) for the crossflow instability. In particular, we now appreciate a more complete view of the dependence of the vortex waveangle on $\psi$ from that outlined in figure 3. In our previous study, theoretical results (both numerical and asymptotic) based on a crossflow instability showed significant departure from the experimental measurements of Kobayashi \& Izumi (1983) for small values of $\psi$, thereby motivating the present investigation. Formulating the appropriate centrifugal instability analyses has not only confirmed the existence of the centrifugal modes, but also identified the parameter regime in which such modes play a dominant role in the instability characteristics of the flow, for $\psi \leq 45^{\circ}$ and $0^{\circ} \leq \phi \leq 10^{\circ}$. A major finding of both the numerical and asymptotic studies in this parameter regime is that the centrifugal modes compare well with the experiments of Kobayashi \& Izumi (1983), filling the missing region where up to now the crossflow instability theory presented in Garrett et al. (2009) was insufficient.

Furthermore, in Garrett et al. (2009), we hypothesised the existence of a potential 'critical' cone half-angle, either side of which the various instability modes dominated. Indeed, from figure 10, we see that combining results from the crossflow instability analysis of Garrett et al. (2009) (at critical Reynolds numbers), the current centrifugal instability analysis and the experimental measurements of Kobayashi \& Izumi (1983), the measurements appear to converge in a triangular region of parameter space centered around $\psi=40^{\circ}$. Hence, the region around this value appears to be where the change of dominant instability occurs.

However, importantly, it must be noted that in some cases both co-rotating and counter-rotating vortices have been observed on rotating cones with small $\psi$, for example, as discussed in Kohama (2000). This suggests that both instabilities may be present for certain set-ups and the change of instability is in fact a gradual process, rather than being fixed at one particular $\psi$. Nevertheless, further investigation, in particular experimental measurements around this sensitive region of parameter space would be highly recommended to shed further light on the flow characteristics; for example pertaining to how the instability mechanisms might interact and lead to transition to turbulent flow. In addition, a complete numerical solution to equations (A 1)-(A 4), may reveal more information about the relative importance of the stability modes, which would help to determine which are the most dangerous modes in various parameter regimes. 
Secondly, it is worth noting that the scope of the current study is limited to a linear analysis, concerned only with convective instability. More importantly, the study considers perturbations that are stationary with respect to the rotating cone. While it is known that non-stationary travelling disturbances are the most unstable modes for rotating-disk flows (see for example Corke \& Knasiak (1998) and Hussain et al. (2011)), stationary disturbances are the most easily observed in experiments, as they are excited by surface roughness elements. Combined with the current analysis, such studies warrant further investigation into whether travelling modes play as important a role for rotating cones with much slender half-angles.

Finally, while both the current study and Garrett et al. (2009) consider rotating cones in still fluid, the question of how introducing external parameters, such as an enforced axial flow, affects the interplay between the dominant instability modes remains. The crossflow instability for broader rotating cones in axial flow has been considered and discussed in Garrett et al. (2010). Meanwhile, an investigation into the centrifugal instability for more slender rotating cones within an imposed axial flow is currently underway and we hope to report on this in the near future.

\section{Acknowledgments}

This work was partly supported by the EPSRC [grant number EP/G061637/1]. SJG wishes to acknowledge useful discussions with the late Dr Tim Nickels prior to his untimely death in 2010. Study leave from the University of Leicester and the kind hospitality of KTH and NORDITA, Stockholm, where much of this paper was drafted, are also acknowledged.

\section{Appendix A. The governing perturbation equations}

$$
\begin{aligned}
& \mathrm{i} a \frac{\sin \psi}{\bar{h}_{1}} \tilde{u}+\frac{\sin \psi(\tilde{u} \cos \phi+\tilde{v} \sin \phi)}{\bar{h}_{1} \bar{h}_{2}}+\mathrm{i} b \frac{\sin \psi}{\bar{h}_{2}} \tilde{v}+\frac{\partial \tilde{w}}{\partial \eta}+\left(\frac{\cos \psi \cos ^{2} \phi}{\bar{h}_{2}}+\frac{\cos \psi \sin ^{2} \phi}{\bar{h}_{1}}\right) \tilde{w}=0 \\
& \left(\mathrm{i} a \frac{(1+\check{x} \cos \phi-\bar{y} \sin \phi)}{\bar{h}_{1}} \tilde{U}+\mathrm{i} b \frac{(1+\check{x} \cos \phi-\bar{y} \sin \phi)}{\bar{h}_{2}} \tilde{V}+W \frac{\partial}{\partial \eta}\right) \tilde{u} \\
& +\frac{\tilde{U} \cos \phi}{\bar{h}_{1}} \tilde{u}-\frac{\tilde{U} \sin \phi}{\bar{h}_{2}} \tilde{v}+\frac{(1+\check{x} \cos \phi-\bar{y} \sin \phi)}{\sin \psi} \frac{\partial \tilde{U}}{\partial \eta} \tilde{w} \\
& +\frac{(1+\check{x} \cos \phi-\bar{y} \sin \phi)}{\bar{h}_{1} \bar{h}_{2}}(\tilde{U} \tilde{v}+\tilde{V} \tilde{u}) \sin \phi \\
& +\left(\frac{(1+\check{x} \cos \phi-\bar{y} \sin \phi)}{\bar{h}_{1}} \tilde{U} \tilde{w}+\frac{\sin \psi}{\bar{h}_{1}} W \tilde{u}\right) \cot \psi \sin ^{2} \phi \\
& -2 \frac{(1+\check{x} \cos \phi-\bar{y} \sin \phi)}{\bar{h}_{1} \bar{h}_{2}} \tilde{V} \tilde{v} \cos \phi-2(\tilde{w} \cot \psi \sin \phi+\tilde{v}) \\
& =-\mathrm{i} a \frac{\sin \psi}{\bar{h}_{1}} \tilde{p}+\bar{\nabla}^{2} \tilde{u}+\left[\frac{\partial}{\partial \check{x}}\left(\frac{1}{\bar{h}_{1} \bar{h}_{2}} \frac{\partial\left(\bar{h}_{1} \bar{h}_{2}\right)}{\partial \check{x}}\right) \tilde{u}-2 \mathrm{i} b \frac{\sin \psi}{\bar{h}_{2}^{2}} \frac{\partial \bar{h}_{2}}{\partial \check{x}} \tilde{v}\right. \\
& \left.-\left(\frac{\sin \psi}{\bar{h}_{2}^{3}} \frac{\partial \bar{h}_{2}}{\partial \bar{y}} \frac{\partial \bar{h}_{2}}{\partial \check{x}}+\frac{\sin \psi}{\bar{h}_{1}^{2} \bar{h}_{2}} \frac{\partial \bar{h}_{1}}{\partial \bar{y}} \frac{\partial \bar{h}_{1}}{\partial \check{x}}\right) \tilde{v}-\left(\frac{\sin \psi}{\bar{h}_{1}^{3}} \frac{\partial \bar{h}_{1}}{\partial \bar{\eta}} \frac{\partial \bar{h}_{1}}{\partial \check{x}}+\frac{\sin \psi}{\bar{h}_{1} \bar{h}_{2}^{2}} \frac{\partial \bar{h}_{2}}{\partial \bar{\eta}} \frac{\partial \bar{h}_{2}}{\partial \check{x}}\right) \tilde{w}\right],
\end{aligned}
$$




$$
\begin{aligned}
& \left(\mathrm{i} a \frac{(1+\check{x} \cos \phi-\bar{y} \sin \phi)}{\bar{h}_{1}} \tilde{U}+\mathrm{i} b \frac{(1+\check{x} \cos \phi-\bar{y} \sin \phi)}{\bar{h}_{2}} \tilde{V}+W \frac{\partial}{\partial \eta}\right) \tilde{v} \\
& +\frac{\tilde{V} \cos \phi}{\bar{h}_{1}} \tilde{u}-\frac{\tilde{V} \sin \phi}{\bar{h}_{2}} \tilde{v}+\frac{(1+\check{x} \cos \phi-\bar{y} \sin \phi)}{\sin \psi} \frac{\partial \tilde{V}}{\partial \eta} \tilde{w} \\
& +\frac{(1+\check{x} \cos \phi-\bar{y} \sin \phi)}{\bar{h}_{1} \bar{h}_{2}}(\tilde{U} \tilde{v}+\tilde{V} \tilde{u}) \cos \phi \\
& +\left(\frac{(1+\check{x} \cos \phi-\bar{y} \sin \phi)}{\bar{h}_{2}} \tilde{V} \tilde{w}+\frac{\sin \psi}{\bar{h}_{2}} W \tilde{v}\right) \cot \psi \cos ^{2} \phi \\
& -2 \frac{(1+\check{x} \cos \phi-\bar{y} \sin \phi)}{\bar{h}_{1} \bar{h}_{2}} \tilde{U} \tilde{u} \sin \phi+2(\tilde{w} \cot \psi \cos \phi+\tilde{u}) \\
& =-\mathrm{i} b \frac{\sin \psi}{\bar{h}_{2}} \tilde{p}+\bar{\nabla}^{2} \tilde{v}+\left[\left(\frac{\sin \psi}{\bar{h}_{2}^{3}} \frac{\partial \bar{h}_{2}}{\partial \check{x}} \frac{\partial \bar{h}_{2}}{\partial \bar{y}}+\frac{\sin \psi}{\bar{h}_{1}^{2} \bar{h}_{2}} \frac{\partial \bar{h}_{1}}{\partial \check{x}} \frac{\partial \bar{h}_{1}}{\partial \bar{y}}\right) \tilde{u}\right. \\
& +\frac{\sin ^{2} \psi}{\bar{h}_{2}^{2}} \tilde{v}-2 \mathrm{i} b \frac{\sin \psi}{\bar{h}_{2}^{2}}\left(\frac{\partial \bar{h}_{2}}{\partial \check{x}} \tilde{u}+\frac{\partial \bar{h}_{2}}{\partial \bar{\eta}} \tilde{w}\right) \\
& \left.-\left(\frac{\sin \psi}{\bar{h}_{1}^{3}} \frac{\partial \bar{h}_{1}}{\partial \bar{\eta}} \frac{\partial \bar{h}_{1}}{\partial \bar{y}}+\frac{\sin \psi}{\bar{h}_{1} \bar{h}_{2}^{2}} \frac{\partial \bar{h}_{2}}{\partial \bar{\eta}} \frac{\partial \bar{h}_{2}}{\partial \bar{y}}\right) \tilde{w}\right] \\
& \left(\mathrm{i} a \frac{(1+\check{x} \cos \phi-\bar{y} \sin \phi)}{\bar{h}_{1}} \tilde{U}+\mathrm{i} b \frac{(1+\check{x} \cos \phi-\bar{y} \sin \phi)}{\bar{h}_{2}} \tilde{V}+W \frac{\partial}{\partial \eta}\right) \tilde{w}+\frac{\partial W}{\partial \eta} \tilde{w} \\
& -2 \frac{(1+\check{x} \cos \phi-\bar{y} \sin \phi)}{\bar{h}_{1}} \tilde{U} \tilde{u} \cot \psi \sin ^{2} \phi-2 \frac{(1+\check{x} \cos \phi-\bar{y} \sin \phi)}{\bar{h}_{2}} \tilde{V} \tilde{v} \cot \psi \cos ^{2} \phi \\
& -2(\tilde{v} \cot \psi \cos \phi-\tilde{u} \cot \psi \sin \phi)=-\frac{\partial \tilde{p}}{\partial \eta}+\bar{\nabla}^{2} \tilde{w} \\
& -\left(\frac{\sin \psi}{\bar{h}_{1}^{3}} \frac{\partial \bar{h}_{1}}{\partial \check{x}} \frac{\partial \bar{h}_{1}}{\partial \eta}+\frac{\sin \psi}{\bar{h}_{1} \bar{h}_{2}^{2}} \frac{\partial \bar{h}_{2}}{\partial \check{x}} \frac{\partial \bar{h}_{2}}{\partial \bar{\eta}}\right) \tilde{u} \\
& +\left(\frac{\sin \psi}{\bar{h}_{2}^{3}} \frac{\partial \bar{h}_{2}}{\partial \bar{y}} \frac{\partial \bar{h}_{2}}{\partial \bar{\eta}}+\frac{\sin \psi}{\bar{h}_{1}^{2} \bar{h}_{2}} \frac{\partial \bar{h}_{1}}{\partial \bar{y}} \frac{\partial \bar{h}_{1}}{\partial \eta}\right) \tilde{v} \\
& +2\left(\frac{\sin \psi}{\bar{h}_{1}^{2}} \frac{\partial \bar{h}_{1}}{\partial \eta} \frac{\partial \tilde{u}}{\partial \check{x}}+\frac{\sin \psi}{\bar{h}_{2}^{2}} \frac{\partial \bar{h}_{2}}{\partial \eta} \frac{\partial \tilde{v}}{\partial \bar{y}}\right)-\frac{\partial}{\partial \eta}\left(\frac{1}{\bar{h}_{1} \bar{h}_{2}} \frac{\partial\left(\bar{h}_{1} \bar{h}_{2}\right)}{\partial \eta}\right) \tilde{w},
\end{aligned}
$$

where $\bar{\nabla}^{2}=\mathrm{R}^{-1} \nabla^{2}$ is now the non-dimensional re-scaled Laplacian operator in the logarithmic spiral wave coordinate setup, which may be expressed as

$\left.\bar{\nabla}^{2}=\frac{\partial^{2}}{\partial \eta^{2}}-\left(a^{2} \frac{\sin ^{2} \psi}{\bar{h}_{1}^{2}}+b^{2} \frac{\sin ^{2} \psi}{\bar{h}_{2}^{2}}\right)+\frac{\sin ^{2} \psi}{\bar{h}_{1} \bar{h}_{2}}\left[\mathrm{i} a \frac{\partial}{\partial \check{x}}\left(\frac{\bar{h}_{2}}{\bar{h}_{1}}\right)+\mathrm{i} b \frac{\partial}{\partial \bar{y}}\left(\frac{\bar{h}_{1}}{\bar{h}_{2}}\right)+\frac{\partial}{\partial \eta}\left(\bar{h}_{1} \bar{h}_{2}\right) \frac{\partial}{\partial \eta}\right).\right]$

The scale factors are given by

$$
\begin{aligned}
& h_{1}=\frac{\bar{h}_{1}}{\sin \psi}+O\left(\mathrm{R}^{-\frac{1}{2}}\right), \\
& h_{2}=\frac{\bar{h}_{2}}{\sin \psi}+O\left(\mathrm{R}^{-\frac{1}{2}}\right),
\end{aligned}
$$

where

$$
\begin{aligned}
& \bar{h}_{1}=1+\check{x} \cos \phi-\bar{y} \sin \phi+\eta \cos \psi \sin ^{2} \phi, \\
& \bar{h}_{2}=1+\check{x} \cos \phi-\bar{y} \sin \phi+\eta \cos \psi \cos ^{2} \phi .
\end{aligned}
$$




\section{REFERENCES}

Corke, T. C. \& Knasiak, K. F. 1998 'Stationary traveling cross-flow mode interactions on a rotating disk' J. Fluid Mech. 355, 285-315.

Davies, C. \& CARPenter, P. W. 2003 Global behaviour corresponding to the absolute instability of the rotating-disk boundary layer. J. Fluid Mech. 486, 287-329.

Denier, J. P., Hall, P. \& Seddougui, S. O. 1991 'On the receptivity problem for Görtler vortices: vortex motions induced by wall roughness.' Phil. Trans. R Soc. Lond. A 335, $51-85$.

Garrett, S. J. \& Peake, N. 2007 'The absolute instability of the boundary layer on a rotating cone.' European. J. Mech. B. 26, 344-53.

Garrett, S. J., Hussain, Z. \& Stephen, S. O. 2009 'The crossflow instability of the boundary layer on a rotating cone.' J. Fluid Mech. 622, 209-232.

Garrett, S. J., Hussain, Z. \& Stephen, S. O. 2010 'Boundary-layer transition on broad cones rotating in an imposed axial flow.' AIAA Journal, 48, No. 6., 1184-1194.

Garrett, S. J. 2010 'Linear growth rates of type I \& II convective modes within the rotatingcone boundary layer.' Fluid Dyn. Res., 42, 025504.

Gregory, N., Stuart, J. T. \& Walker, W. S. 1955 'On the stability of three-dimensional boundary layers with application to the flow due to a rotating disk.' Phil. Trans. R. Soc. Lond. A 248, 155-99.

HALL, P. 1982 'Taylor-Görtler vortices in fully developed or boundary-layer flows: linear theory.' J. Fluid Mech. 124, 475-94.

Healey, J.J. 2010 Model for unstable global modes in the rotating-disk boundary layer. $J$. Fluid Mech., 663, 148-159.

Hussain, Z. 2010 'Stability and transition of three-dimensional rotating boundary layers.' PhD thesis, University of Birmingham.

Hussain, Z., Garrett, S. J. \& Stephen, S. O. 2011 'The convective instability of the boundary layer on a rotating disk in axial flow.' Phys. Fluids 23, 1141108

Hussain, Z., Stephen, S.O. \& Garrett, S.J. 2012 'The centrifugal instability of a slender rotating cone', Journal of Algorithms \& Computational Technology, Vol. 6, No. 1.

Imayama, S., Alfredsson, P.H. \& Lingwood, R.J. 2013 An experimental study of edge effects on rotating-disk transition. J. Fluid Mech., 716, 638-657.

Kappesser, R., Greif, R. \& Cornet, I. 1973 'Mass transfer on rotating cones.' Appl. Sci. Res. 28, 442-52.

KoBAyASHI, R. 1981 'Linear stability theory of boundary layer along a cone rotating in axial flow.' Bull. Japan Soc. Mech. Engrs. 24, 934-940.

Kobayashi, R., Kohama, Y. \& Kurosawa, M. 1983 'Boundary-layer transition on a rotating cone in axial flow.' J. Fluid Mech. 127, 341-52.

Kobayashi, R. \& Izumi, H. 1983 'Boundary-layer transition on a rotating cone in still fluid.' J. Fluid Mech. 127, 353-64.

Kobayashi, R. 1994 'Review: Laminar-to-Turbulent Transition of Three-Dimensional Boundary Layers on Rotating Bodies.' Trans. ASME 116, 200-11.

KoнAma, Y. 1985 'Flow structures formed by axisymmetric spinning bodies.' AIAA J. 23, 1445.

Kohama, Y. P. 2000 'Three-dimensional boundary layer transition study.' Current Science 79(6), 800-7.

Kreith, F., Ellis, D. \& Giesing, J. 1962 'An experimental investigation of the flow engendered by a rotating cone.' Appl. Sci. Res. A11, 430-40.

LingwOOD, R. J. 1995 'Absolute instability of the boundary layer on a rotating disk.' J. Fluid Mech. 299, 17-33.

Lingwood, R. J. 1996 'An experimental study of absolute instability of the rotating-disk boundary layer flow.' J. Fluid Mech. 314, 373-405.

MALIK, M. R. 1986 The neutral curve for stationary disturbances in rotating-disk flow. J. Fluid Mech. 164, 275-87.

PIER, B. 2003 'Finite-amplitude crossflow vortices, secondary instability and transition in the rotating-disk boundary layer' J. Fluid Mech. 487, 315-343.

Reed, H. L. \& SARIC, W. S. 1989 'Stability of three-dimensional boundary layers.' Ann. Rev. Fluid Mech. 21, 235-84. 
Saric, W. S., Reed, H. L. \& White, E. B. 2003 'Stability and transition of three-dimensional boundary layers.' Ann. Rev. Fluid Mech. 35, 413-40.

Towers, P. D. \& Garrett, S. J. 2014 'Similarity solutions of compressible flow over a rotating cone with surface suction.' Thermal Science Online-First (00), 32.

Towers, P. D. \& Garrett, S. J. 'The stability of the compressible boundary-layer flows over rotating cones with surface mass flux.' In preparation. 